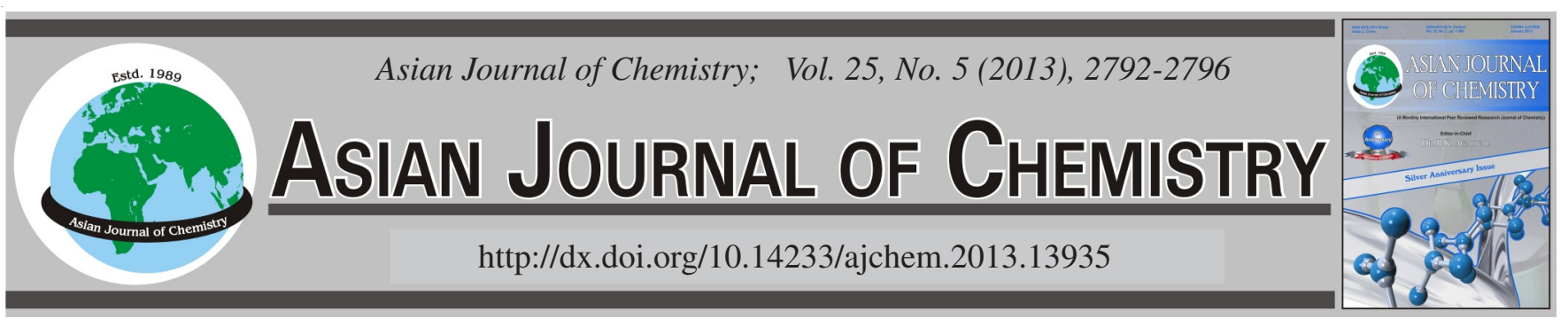

\title{
Oxidative Regeneration of Carbonyl Compounds from Oximes by Pyridinium Fluorochromate: A Kinetic and Mechanistic Study
}

\author{
Ajay Sharma ${ }^{1}$, Neha Vyas ${ }^{1}$, A. Choudhary ${ }^{1}$, PTSRK Prasadrao ${ }^{2}$ and Vinita Sharma ${ }^{1, *}$
}

${ }^{1}$ Chemical Kinetics Laboratory, Department of Chemistry, Jai Narain Vyas University, Jodhpur-342 005, India

${ }^{2}$ Department of Chemistry, PB Siddhartha College of Arts \& Science, Siddhartha Nagar, Moghalraja Puram, Vijayawada-520 010, India

*Corresponding author: E-mail: drvsharma29@gmail.com

(Received: 19 April 2012;

Accepted: 23 November 2012)

AJC-12455

\begin{abstract}
The oxidative deoximination of several aldoximes and ketoximes by pyridinium fluorochromate, in dimethyl sulphoxide, exhibited a first order dependence on pyridinium fluorochromate. A Michaelis-Menten type kinetics was observed with respect to oximes. The oxidation of ketoximes is slower than that of aldoximes. The rates of oxidation of aldoximes correlated well in terms of Pavelich-Taft dual substituentparameter equation. The low positive value of polar reaction constant indicated a nucleophilic attack by a chromate-oxygen on the carbon. The reaction is subjected to steric hindrance by the alkyl groups. The reaction of acetaldoxime has been studied in 19 different organic solvents. The solvent effect has been analyzed by multiparametric equations. A mechanism involving the formation of a cyclic intermediate, in the rate-determining step is suggested.
\end{abstract}

Key Words: Carbonyl compounds, Halochromates, Kinetics, Mechanism, Oxidation, Oximes.

\section{INTRODUCTION}

Regeneration of carbonyl compounds from its derivatives under mild conditions is an important process in synthetic organic chemistry. Several oxidative methods are available for deoximation ${ }^{1}$. Halochromates have been used as mild and selective oxidizing reagents in synthetic organic chemistry ${ }^{2-5}$. Pyridinium fluorochromate is also one such reagent ${ }^{6}$. We have been interested in the kinetics of reactions of complexed $\mathrm{Cr}(\mathrm{VI})$ species and have already published several reports on oxidation aspects of quinolinium ${ }^{7,8}$ and pyridinium ${ }^{9,10}$ halochromates. Therefore in continuation of our earlier work, we report here the kinetics of the oxidative deoximation of several aldo- and keto-oximes by pyridinium fluorochromate in several organic solvents but mainly in dimethyl sulphoxide. Mechanistic aspects have also been discussed.

\section{EXPERIMENTAL}

Oximes were prepared by the reported standard methods ${ }^{11}$ and their mp were checked with the literature values. Pyridinium fluorochromate also was prepared by the reported method $^{6}$. Solvents were purified by the usual procedure ${ }^{12}$.

Product analysis: The oxidation of the oximes results in the regeneration of corresponding carbonyl compounds, as confirmed by TLC (eluent: $\mathrm{CCl}_{4} / \mathrm{Et}_{2} \mathrm{O}$ ). Isolation of the product was attempted in the oxidation of oximes of benzaldehyde and acetophenone. In a typical experiment, the oxime $(0.2$ $\mathrm{mol})$ and pyridinium fluorochromate $(0.02 \mathrm{~mol})$ were dissolved in $50 \mathrm{~mL}$ of dimethyl sulphoxide and allowed to stand for $c a$. $10 \mathrm{~h}$ for the completion of the reaction. Silica gel $(5 \mathrm{~g})$ was then added to the reaction mixture and the mixture was stirred for $15 \mathrm{~min}^{13}$. It was then filtered and the solid residue was washed with the solvent ( $2 \% 15 \mathrm{~mL})$. The solvent was removed on a rotary evaporator and the residue was purified on a silica-gel column (eluent: $\mathrm{CCl}_{4} / \mathrm{Et}_{2} \mathrm{O}$ ). Evaporation of the solvent afforded the pure carbonyl compound. Yields of benzaldehyde and acetophenone were $1.83 \mathrm{~g}(86 \%)$ and 2.11 $\mathrm{g}(88 \%)$ respectively. The presence of $\mathrm{HNO}_{2}$ in completely reduced reaction mixtures was confirmed by a positive starchiodide test ${ }^{14}$. The oxidation state of chromium in a completely reduced reaction mixture, as determined by an iodometric method, is $3.95 \pm 0.15$.

Kinetics measurements: The reactions were studied under pseudo-first-order conditions by keeping a large excess ( $\times 10$ or more) of the oxime over pyridinium fluorochromate. The solvent was dimethyl sulphoxide, unless mentioned otherwise. The reactions were studied at constant temperature $( \pm 0.1 \mathrm{~K})$. The reactions were followed by monitoring the decrease in the concentration of pyridinium fluorochromate at $370 \mathrm{~nm}$ spectrophotometrically. The pseudo-first-order rate constant, $\mathrm{k}_{\mathrm{obs}}$, was evaluated from the linear least-squares plots of $\log$ [PFC] versus time. Duplicate kinetic runs showed the 
rate constants to be reproducible to within $\pm 4 \%$. The second order rate constants were evaluated from the relation $\mathrm{k}_{2}=\mathrm{k}_{\mathrm{obs}} /$ [reductant]. All reactions, other than those to study the effect of $\left[\mathrm{H}^{+}\right]$, were performed in the absence of $\mathrm{TsOH}$.

\section{RESULTS AND DISCUSSION}

Stoichiometry: The analysis of products indicated the following overall reaction.

$\mathrm{R}_{2} \mathrm{C}=\mathrm{N}-\mathrm{OH}+2 \mathrm{CrO}_{2} \mathrm{ClOMH} \rightarrow \mathrm{R}_{2} \mathrm{C}=\mathrm{O}+\mathrm{HNO}_{2}+2 \mathrm{CrOClOMH}(1)$

Thus pyridinium fluorochromate undergoes a two electron change. This is in accord with the earlier observations with other halochromates ${ }^{6-8}$. It has already been shown that both pyridinium fluorochromate ${ }^{15}$ and pyridinium chlorochromate ${ }^{16}$ act as two electron oxidants and are reduced to chromium(IV) species.

Rate laws: The reactions were found to be first order with respect to pyridinium fluorochromate (Fig. 1). In individual kinetic runs, plots of $\log [\mathrm{PFC}]$ versus time were linear $\left(\mathrm{r}^{2}>\right.$ 0.995). Further, it was found that the observed rate constant, $\mathrm{k}_{\mathrm{obs}}$, does not depend on the initial concentration of pyridinium fluorochromate. The order with respect to oxime was less than one (Table-1). A plot of $1 / \mathrm{k}_{\text {obs }}$ versus $1 /$ [Oxime] was linear with an intercept on the rate ordinate (Fig. 2). Thus MichaelisMenten type kinetics were observed with respect to oxime. This leads to the postulation of following overall mechanism (eqns. 2 and 3) and the rate law (4).

$$
\begin{gathered}
\text { Oxime }+ \text { PFC } \stackrel{\mathbf{K}}{\longleftrightarrow}[\text { Complex }] \\
\text { Rate }=\mathrm{k}_{2} \mathrm{~K}[\text { Oxime }][\mathrm{PFC}] /(1+\mathrm{K}[\text { Oxime }])
\end{gathered}
$$

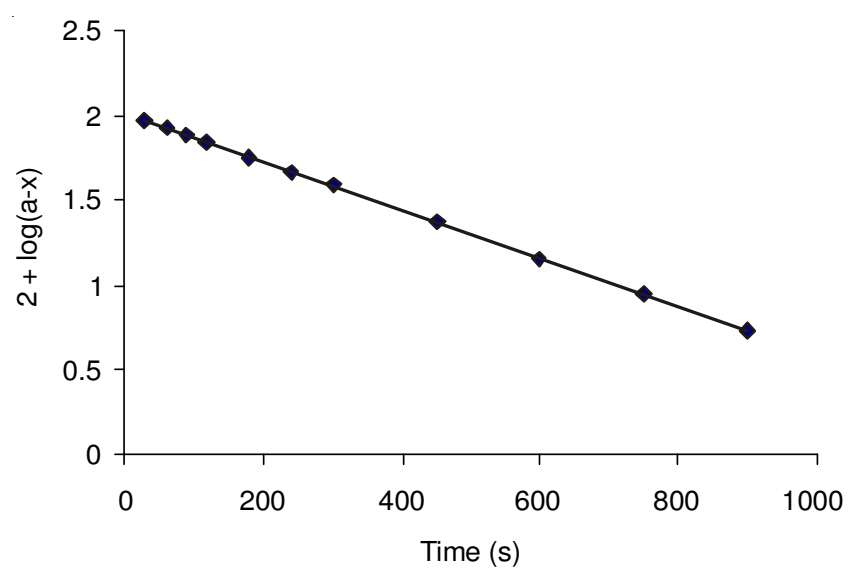

Fig. 1. Oxidation of acetaldoxime by PFC: A typical Kinetic run

The dependence of $\mathrm{k}_{\mathrm{obs}}$ on the concentration of oxime was studied at different temperatures and the values of $\mathrm{K}$ and $\mathrm{k}_{2}$ were evaluated from the double reciprocal plots. The thermodynamic parameters for the complex formation and activation parameters of the disproportionation of the complexes, at $298 \mathrm{~K}$, were calculated from the values of $\mathrm{K}$ and $\mathrm{k}_{2}$ respectively at different temperatures (Tables 2 and 3).

Effect of solvents: The oxidation of acetaldoxime was studied in nineteen different solvents. The choice of solvents was limited due to the solubility of the reactants and the reaction of pyridinium fluorochromate with primary and secondary alcohols. There was no reaction with chosen solvents. The kinetics were similar in all the solvents. The values of $\mathrm{k}_{2}$ are recorded in Table-4.

\begin{tabular}{ccc}
\hline \multicolumn{3}{c}{ TABLE-1 } \\
\multicolumn{3}{|c}{ RATE CONSTANTS FOR THE OXIDATION OF } \\
ACETALDOXIME BY QFC AT 288 K \\
\hline $10^{3}[\mathrm{PFC}]\left(\mathrm{mol} \mathrm{dm}^{-3}\right)$ & [Oxime] $\left(\mathrm{mol} \mathrm{dm}^{-3}\right)$ & $10^{4} \mathrm{k}_{\mathrm{obs}}\left(\mathrm{s}^{-1}\right)$ \\
\hline 1.0 & 0.10 & 11.9 \\
1.0 & 0.20 & 17.5 \\
1.0 & 0.40 & 22.7 \\
1.0 & 0.60 & 25.2 \\
1.0 & 0.80 & 26.7 \\
1.0 & 1.00 & 27.6 \\
1.0 & 1.50 & 29.1 \\
1.0 & 3.00 & 30.6 \\
2.0 & 0.40 & 23.4 \\
4.0 & 0.40 & 21.6 \\
6.0 & 0.40 & 25.5 \\
8.0 & 0.40 & 23.0 \\
1.0 & 0.20 & $18.9^{*}$ \\
\hline
\end{tabular}

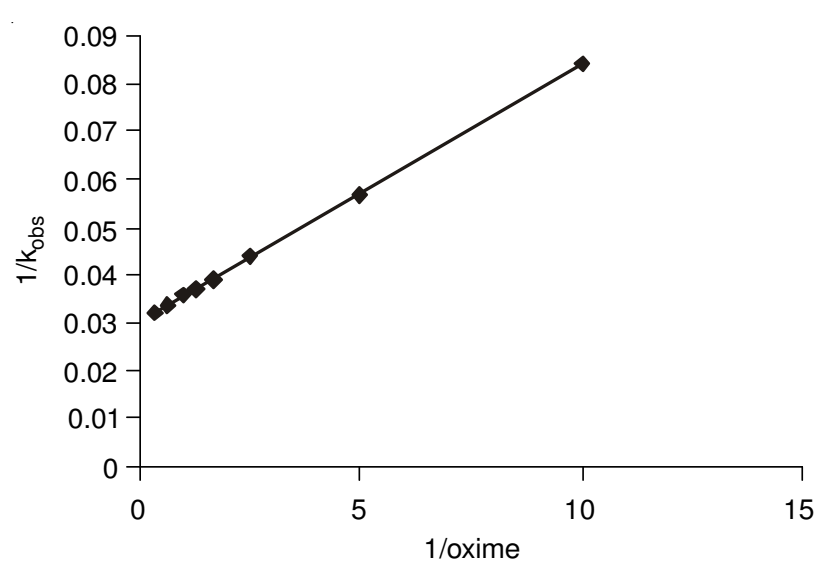

Fig. 2. Oxidation of Acetaldoxime by PFC: A double reciprocal plot

The linear correlation $\left(r^{2}=0.9478\right)$ between the values of the activation enthalpies and entropies of the reaction indicated the operation of a significant compensation effect in this reaction ${ }^{17}$. The reaction exhibited an excellent isokinetic effect (temperature $508 \pm 59 \mathrm{~K}$ ) also, as determined by Exner's method $^{18}$. An Exner's plot between $\log \mathrm{k}_{2}$ at $288 \mathrm{~K}$ and at $318 \mathrm{~K}$ is linear $\left(r^{2}=0.9966\right.$, slope $\left.=0.8048 \pm 0.0236\right)$ (Fig. 3). The value of isokinetic temperature is $567 \pm 67 \mathrm{~K}$. A linear isokinetic relationship is a necessary condition for the validity of linear free energy relationships. It also implies that all reactions so correlated follow a similar mechanism ${ }^{18}$.

Solvent effect: The rate constants, $\mathrm{k}_{2}$, of the oxidation of acetaldoxime in 18 solvents $\left(\mathrm{CS}_{2}\right.$ was not considered, as the complete range of solvent parameters was not available) were correlated in terms of the linear solvation energy relationship (eqn. 2) of Kamlet et al. ${ }^{19}$.

$$
\log \mathrm{k}_{2}=\mathrm{A}_{0}+\mathrm{p} \pi^{*}+\mathrm{b} \beta+\mathrm{a} \alpha
$$

In this equation, $\pi^{*}$ represents the solvent polarity, $\beta$ the hydrogen bond acceptor basicities and $\alpha$ is the hydrogen bond donor acidity. $\mathrm{A}_{0}$ is the intercept term. It may be mentioned here that out of the 18 solvents, 12 have a value of zero for $\alpha$. 
TABLE-2

RATE CONSTANTS AND ACTIVATION PARAMETERS FOR THE OXIDATION OF PFC-OXIMES $\left(\mathrm{R}^{1} \mathrm{R}^{2} \mathrm{C}=\mathrm{N}-\mathrm{OH}\right) \mathrm{COMPLEXES}$

\begin{tabular}{|c|c|c|c|c|c|c|c|c|}
\hline \multirow{2}{*}{\multicolumn{2}{|c|}{ Substituent }} & \multicolumn{4}{|c|}{$10^{4} k_{2}\left(\mathrm{dm}^{3} \mathrm{~mol}^{-1} \mathrm{~s}^{-1}\right)$} & \multirow{2}{*}{$\begin{array}{c}-\Delta \mathrm{H}^{*} \\
\left(\mathrm{~kJ} \mathrm{~mol}^{-1}\right) \\
\end{array}$} & \multirow{2}{*}{$\begin{array}{c}-\Delta \mathrm{S}^{*} \\
\left(\mathrm{~J} \mathrm{~mol}^{-1} \mathrm{~K}^{-1}\right)\end{array}$} & \multirow{2}{*}{$\begin{array}{c}-\Delta \mathrm{G}^{*} \\
\left(\mathrm{~kJ} \mathrm{~mol}^{-1}\right)\end{array}$} \\
\hline & & $288 \mathrm{~K}$ & $298 \mathrm{~K}$ & $308 \mathrm{~K}$ & $318 \mathrm{~K}$ & & & \\
\hline $\mathrm{H}$ & $\mathrm{H}$ & 822 & 1090 & 1350 & 1590 & $14.2 \pm 0.7$ & $216 \pm 2$ & $73.6 \pm 0.6$ \\
\hline $\mathrm{H}$ & $\mathrm{Me}$ & 32.4 & 51.8 & 77.4 & 117 & $29.9 \pm 0.3$ & $189 \pm 1$ & $86.1 \pm 0.2$ \\
\hline $\mathrm{H}$ & $\operatorname{Pr}$ & 12.8 & 21.8 & 34.6 & 55.8 & $35.0 \pm 0.4$ & $179 \pm 1$ & $88.2 \pm 0.3$ \\
\hline $\mathrm{H}$ & $\operatorname{Pr}^{I}$ & 9.50 & 16.2 & 26.5 & 43.2 & $35.8 \pm 0.2$ & $179 \pm 1$ & $89.0 \pm 0.2$ \\
\hline $\mathrm{H}$ & $\mathrm{ClCH}_{2}$ & 44.5 & 63.8 & 86.4 & 126 & $23.5 \pm 0.7$ & $209 \pm 2$ & $85.6 \pm 0.5$ \\
\hline $\mathrm{Me}$ & $\mathrm{Me}$ & 3.24 & 5.10 & 7.83 & 12.6 & $31.7 \pm 0.7$ & $202 \pm 2$ & $91.8 \pm 0.5$ \\
\hline Me & Et & 2.58 & 3.96 & 6.12 & 9.36 & $30.2 \pm 0.4$ & $209 \pm 1$ & $92.4 \pm 0.3$ \\
\hline Et & Et & 2.07 & 3.24 & 4.95 & 7.65 & $30.6 \pm 0.4$ & $210 \pm 1$ & $92.9 \pm 0.3$ \\
\hline $\mathrm{Me}$ & $\mathrm{Ph}$ & 5.75 & 9.30 & 15.3 & 23.4 & $33.3 \pm 0.3$ & $192 \pm 1$ & $90.3 \pm 0.2$ \\
\hline
\end{tabular}

TABLE-3

FORMATION CONSTANTS AND THERMODYNAMIC PARAMETERS OF THE OXIDATION OF PFC- OXIMES $\left(\mathrm{R}^{1} \mathrm{R}^{2} \mathrm{C}=\mathrm{N}-\mathrm{OH}\right)$ COMPLEXES

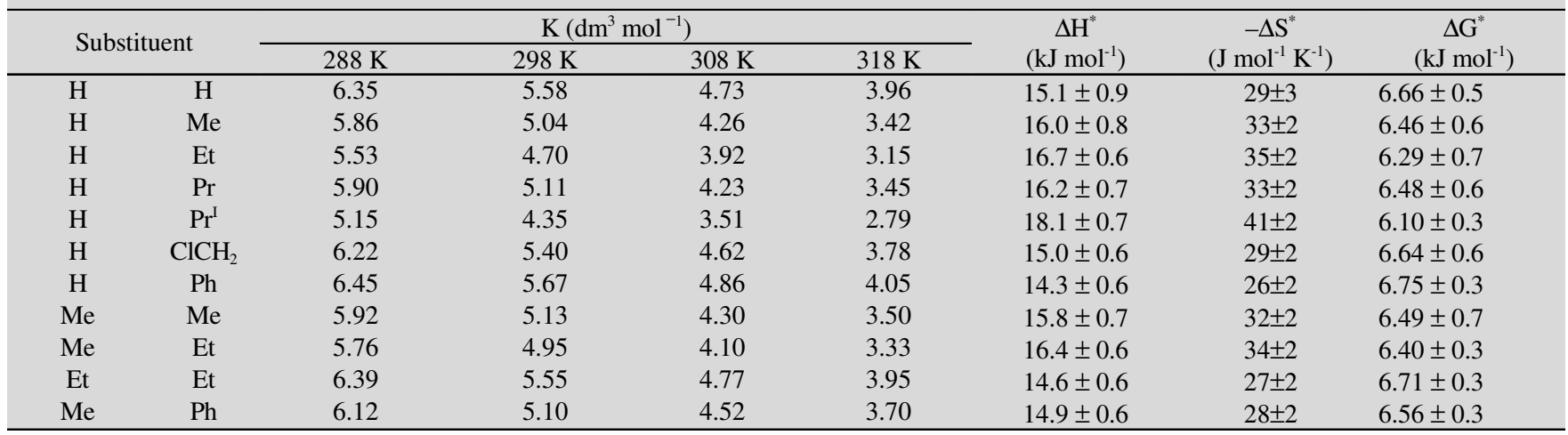

\begin{tabular}{lcc}
\multicolumn{4}{c}{ TABLE-4 } \\
\multicolumn{3}{c}{$\begin{array}{c}\text { EFFECT OF SOLVENTS ON THE OXIDATION OF } \\
\text { ACETALDOXIME BY PFC AT 298 K }\end{array}$} \\
\hline Solvents & $\mathrm{K}\left(\mathrm{dm}^{3} \mathrm{~mol}^{-1}\right)$ & $10^{5} k_{2}$ \\
\hline Chloroform & 5.44 & 45.7 \\
1,2-Dichloroethane & 5.45 & 42.7 \\
Dichloromethane & 5.94 & 35.5 \\
DMSO & 3.42 & 117 \\
Acetone & 4.76 & 33.9 \\
DMF & 5.85 & 63.1 \\
Butanone & 5.55 & 21.9 \\
Nitrobenzene & 3.92 & 39.8 \\
Benzene & 5.45 & 11.7 \\
Cyclohexane & 5.63 & 0.85 \\
Toluene & 4.60 & 7.94 \\
Acetophenone & 5.24 & 38.9 \\
THF & 5.59 & 14.8 \\
t-butylalcohol & 4.76 & 20.9 \\
1,4-Dioxane & 5.45 & 17.4 \\
1,2-Dimethoxyethane & 4.33 & 9.77 \\
CS & 5.51 & 4.47 \\
Acetic acid & 4.59 & 19.1 \\
Ethyl acetate & 5.13 & 12.1 \\
\hline
\end{tabular}

The results of correlation analyses in terms of eqn. (2), a biparametric equation involving $\pi^{*}$ and $\beta$ and separately with $\pi^{*}$ and $\beta$ are given below [eqs. (3)-(6)].

$\log k_{2}=-3.93+1.77( \pm 0.21) \pi^{*}+0.15( \pm 0.17) \beta+0.34( \pm 0.16) \alpha(3)$

$$
\mathrm{R}^{2}=0.8615 ; \mathrm{sd}=0.19 ; \mathrm{n}=18 ; \psi=0.41
$$

$\log \mathrm{k}_{2}=-3.85+1.65( \pm 0.22) \pi^{*}+0.27( \pm 0.17) \beta$

$$
\mathrm{R}^{2}=0.8189 ; \mathrm{sd}=0.21 ; \mathrm{n}=18 ; \psi=0.45
$$$$
\log \mathrm{k}_{2}=-3.80+1.72( \pm 0.22) \pi^{*}
$$

$$
\begin{gathered}
r^{2}=0.7918 ; s d=0.22 ; n=18 ; \psi=0.47 \\
\quad \log k_{2}=-2.89+0.56( \pm 0.37) \beta \\
r^{2}=0.1260 ; s d=0.44 ; n=18 ; \psi=0.96
\end{gathered}
$$

Here $\mathrm{n}$ is the number of data points and $\psi$ is Exner's statistical parameter ${ }^{20}$.

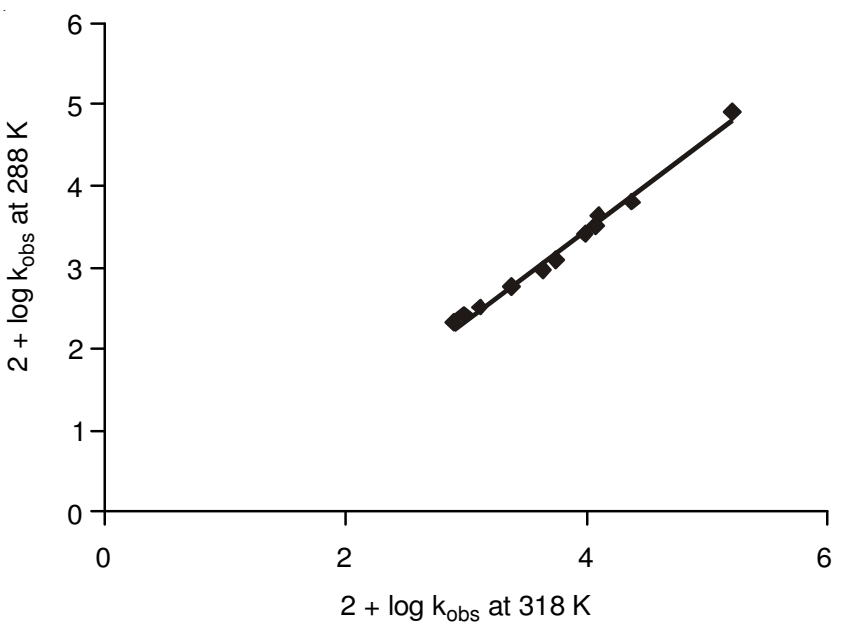

Fig. 3. Exner's isokinetic relationship in the oxidation of oximes by PFC

Kamlet et al. ${ }^{19}$ triparametric equation explains ca. $86 \%$ of the effect of solvent on the oxidation. However, by Exner's criterion $^{18}$ the correlation is not even satisfactory ( $c f$. eqn. 3 ). The major contribution is of solvent polarity. It alone accounted for $c a .79 \%$ of the data. Both $\beta$ and $\alpha$ play relatively minor roles. 
The data on the solvent effect were analyzed in terms of Swain's equation ${ }^{21}$ of cation- and anion-solvating concept of the solvents also [eqn. (7)].

$$
\log \mathrm{k}_{2}=\mathrm{aA}+\mathrm{bB}+\mathrm{C}
$$

Here $\mathrm{A}$ represents the anion-solvating power of the solvent and $\mathrm{B}$ the cation-solvating power. $\mathrm{C}$ is the intercept term. $\mathrm{A}+$ $\mathrm{B})$ is postulated to represent the solvent polarity. The rates in different solvents were analyzed in terms of eqn. (7), separately with $\mathrm{A}$ and $\mathrm{B}$ and with $(\mathrm{A}+\mathrm{B})$.

$$
\begin{aligned}
\log \mathrm{k}_{2}= & 1.33( \pm 0.05) \mathrm{A}+1.64( \pm 0.04) \mathrm{B}-3.86 \\
\mathrm{R}^{2}= & 0.9937 ; \mathrm{sd}=0.04 ; \mathrm{n}=19 ; \psi=0.08 \\
& \log \mathrm{k}_{2}=1.10( \pm 0.54) \mathrm{A}-3.02 \\
\mathrm{r}^{2}= & 0.1937 ; \mathrm{sd}=0.44 ; \mathrm{n}=19 ; \psi=0.92 \\
& \log \mathrm{k}_{2}=1.54( \pm 0.24) \mathrm{B}-3.71 \\
\mathrm{r}^{2}= & 0.7120 ; \mathrm{sd}=0.26 ; \mathrm{n}=19 ; \psi=0.55 \\
& \log \mathrm{k}_{2}=1.53 \pm 0.05(\mathrm{~A}+\mathrm{B})-3.87 \\
& \mathrm{r}^{2}=0.9826 ; \mathrm{sd}=0.06 ; \mathrm{n}=19 ; \psi=0.14
\end{aligned}
$$

The rates of oxidation of acetaldehyde in different solvents showed an excellent correlation in Swain's equation [ $c f$. eqn. (8)] with both the anion- and cation-solvating powers playing almost equal role. However, individually $\mathrm{A}$ and $\mathrm{B}$ are able to account for only 19 and $71 \%$ of the data only. The solvent polarity, represented by $(A+B)$, also exhibited an excellent correlation. In view of the fact that solvent polarity is able to account for $\mathrm{ca} .98 \%$ of the data, an attempt was made to correlate the rate with the relative permittivity of the solvent. However, a plot of $\log \mathrm{k}_{2}$ against the inverse of the relative permittivity is not very significant $\left(\mathrm{r}^{2}=0.4604\right.$; $\mathrm{sd}=0.36 ; \psi=$ $0.75)$. The analysis of solvent effect indicated the formation of an activated complex which is more polar than the reactants. The rate is affected by the solvent polarity.

Correlation analysis of reactivity: We could not find any report about the mechanism of the reaction between a $\mathrm{C}=\mathrm{N}$ bond and a halochromate derivative. However, the reaction of alkenes with chromium(VI) has been well studied ${ }^{22}$. Since, olefinic bonds are not usually subject to a nucleophilic attack, it has been suggested that in the alkene-chromate reaction, an organometallic derivative is formed initially ${ }^{22}$. The organometallic derivative then changes to a chromium(IV) diester in the rate-determining step. However, carbon-nitrogen double bonds, being dipolar in nature, can be easily attacked by a nucleophile. The data in Table- 2 showed that the rate of oxidation of ketoximes is much less as compared to that of the aldoximes. The reason for the slower reaction of ketoximes must be steric. As the central carbon changes from a trigonal to a tetragonal state, the crowding around it increases. This increase in the steric crowding will be more in the case of ketoximes as compared to that in aldoximes. This observation is supported by the correlation analysis of the reactivity of the aldoximes also. The rate of oxidation of the aliphatic oximes did not yield significant correlation separately with Tafts's $\sigma^{*}$ and $E_{S}$ values [eqns. (12) and (13)]. The rates were, therefore, correlated with Pavelich-Taft's ${ }^{23}$ dual substituent-parameter eqn. (14).

$$
\begin{gathered}
\log \mathrm{k}_{2}=0.68 \pm 0.58 \sigma^{*}-2.34 \\
\mathrm{r}^{2}=0.2587, \mathrm{sd}=0.63, \mathrm{n}=6, \psi=0.94, \text { Temp. }=298 \mathrm{~K} \\
\log \mathrm{k}_{2}=1.02 \pm 0.11 \mathrm{E}_{\mathrm{S}}-2.23 \\
\mathrm{r}^{2}=0.9539, \mathrm{sd}=0.16, \mathrm{n}=6, \psi=0.24, \text { Temp. }=298 \mathrm{~K} \\
\log \mathrm{k}_{2}=\rho^{*} \sigma^{*}+\delta \mathrm{E}_{\mathrm{S}}+\log \mathrm{k}_{0}
\end{gathered}
$$

The rates exhibited excellent correlations in terms of the Pavelich-Taft equation (Table-4); the reaction constants (Table5) are being positive.

TABLE-5

REACTION CONSTANTS FOR THE OXIDATIVE DEOXIMINATION OF ALIPHATIC ALDOXIMES BY PFC ${ }^{\mathrm{a}}$

\begin{tabular}{cccccc}
\hline Temp./K & $\rho^{*}$ & $\delta$ & $\mathrm{R}^{2}$ & $\mathrm{sd}$ & $\psi$ \\
\hline 288 & $0.36 \pm 0.01$ & $0.99 \pm 0.01$ & 0.9999 & 0.003 & 0.01 \\
298 & $0.30 \pm 0.02$ & $0.95 \pm 0.02$ & 0.9998 & 0.002 & 0.02 \\
308 & $0.25 \pm 0.01$ & $0.90 \pm 0.01$ & 0.9999 & 0.003 & 0.01 \\
318 & $0.22 \pm 0.01$ & $0.83 \pm 0.02$ & 0.9989 & 0.002 & 0.04 \\
\hline \multicolumn{7}{l}{ Number of compounds is 6}
\end{tabular}

Mechanism: The low positive polar reaction constant points to an almost cyclic transition state in which the formation of the bond between chromate-oxygen and the carbon is somewhat ahead of the formation of $\mathrm{N}-\mathrm{O}$ bond. This supports a nucleophilic attack by a chromate-oxygen on the carbon. The positive steric reaction constant points to a steric hindrance by the substituents. Therefore, the following mechanism (Scheme-I) is proposed for the reaction. The mechanism is supported by the values of activation parameters also. The low values of enthalpy of activation indicate that the bond-cleavage and bond-formation are almost synchronous. The large negative entropies of activation support the formation of a rigid cyclic activated complex from two acyclic molecules.

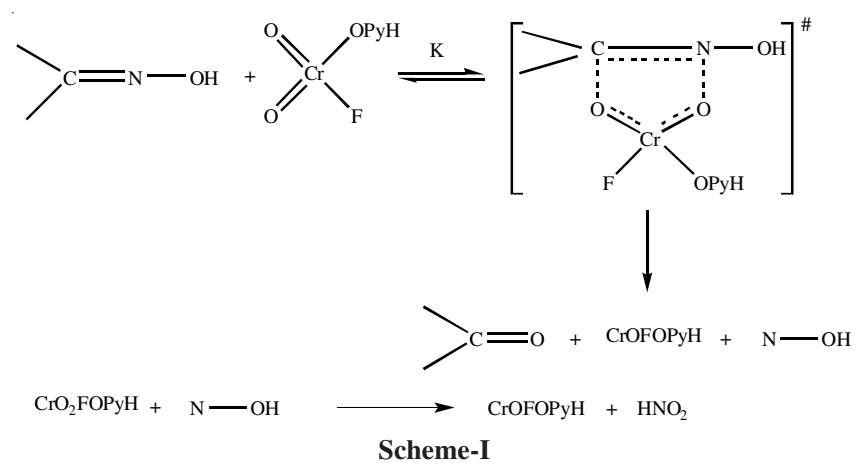

The faster oxidation of benzaldoxime may be attributed to the resonance stablization of the cyclic activated complex. The oxidation of benzphenoxime is much slower. This may well be due to steric hindrance by the bulky phenyl and methyl groups. Hydroxynitrene $(\mathrm{N}-\mathrm{OH})$ has been recently reported as a very reactive intermediate ${ }^{24}$.

\section{ACKNOWLEDGEMENTS}

Thanks are due to University Grants Commission, New Delhi for financial support in the form of Major Research Project and to Professor KK Banerji for his critical suggestions and helpful discussions.

\section{REFERENCES}

1. H. Firouzabadi and A. Sadarian, Synth. Commun., 13, 863 (1983); J. Drabowicz, Synthesis, 125 (1980); P.B. Hopkins, S. Kim, S. Yoo, K.P. Nambiar and J.R. Flack, J. Am. Chem. Soc., 101, 7131 (1979).

2. E.J. Corey and J.W. Suggs, Tetrahedron Lett., 16, 2647 (1975).

3. F.S. Guziec and F.A. Luzio, Synthesis, 691 (1980). 
4. A. Pandurangan, V. Murugesan and M. Palanichamy, J. Indian Chem. Soc., 72, 479 (1995)

5. K. Balasubramanian and V. Prathiba, Indian J. Chem., 25B, 326 (1986).

6. M.N. Bhattacharjee, M.K. Choudhuri, H.S. Dasgupta, N. Roy and D.T. Khathing, Synthesis, 588 (1982).

7. I. Dave, V. Sharma and K.K. Banerji, Indian J. Chem., 39A, 728 (2000).

8. I. Dave, V. Sharma and K.K. Banerji, Indian J. Chem., 41A, 493 (2002).

9. S. Saraswat, V. Sharma and K.K. Banerji, Proc. Indian Acad. Sci., Chem. Sci., 115, 75 (2003).

10. R. Kumbhat and V. Sharma, J. Indian Chem. Soc., 81, 745 (2004).

11. A.I. Vogel, A Text Book of Practical Organic Chemistry, ELBS, London, p. 343 (1973).

12. D.D. Perrin, L. Armarego and D.R. Perrin, Purification of Organic Compounds, Pergamon, Oxford (1966)

13. I.M. Baltrok, M.M. Sadegi, N. Mahmoodi and B. Kharamesh, Indian J. Chem., 36B, 438 (1997).

14. F. Feigl and V. Anger, Spot Tests in Organic Analysis, Elsevier Publication (1966).
15. H.C. Brown, G.C. Rao and S.U. Kulkarni, J. Org. Chem., 44, 2809 (1979).

16. M.N. Bhattacharjee, M.K. Choudhuri and S. Purakayastha, Tetrahedron, 43, 5389 (1987).

17. L. Liu and O.X. Guo, Chem. Rev., 101, 673 (2001).

18. O. Exner, Prog. Phys. Org. Chem., 10, 411 (1973).

19. M.J. Kamlet, J.L.M. Abboud, M.H. Abraham and R.W. Taft, J. Org. Chem., 48, 2877 (1983) and references cited therein.

20. O. Exner, Coll. Chem. Czech. Commun., 31, 3222 (1966).

21. C.G. Swain, M.S. Swain, A.L. Powell and S. Alumni, J. Am. Chem. Soc., 105, 502 (1973).

22. F. Freeman, C.O. Fuselier, C.R. Armstead, C.E. Dalton, P.A. Davidson, E. Karchefski, D.F. Krochman, M.N. Johnson and N.K. Jones, J. Am. Chem. Soc., 103, 1154 (1983).

23. W.A. Pavelich and R.W. Taft, J. Am. Chem. Soc., 79, 4935 (1957).

24. G. Maier, H.P. Reisenauer and M.D. Marco, Angew. Chem. Int. Ed., 38, 108 (1999). 